\title{
Study of light nuclei by polarization observables in electron scattering
}

\author{
Simon Širca \\ Faculty of Mathematics and Physics, University of Ljubljana, Slovenia \\ Jožef Stefan Institute, Ljubljana, Slovenia

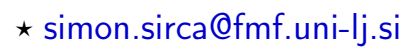
\section{Surrey, UK, 2-6 September 2019 \\ doi:10.21468/SciPostPhysProc.3} \\ Proceedings for the 24th edition of European Few Body Conference,
}

\begin{abstract}
Electron-induced proton, neutron and deuteron knock-out remains the most versatile probe of the electro-magnetic properties and spin structure of light nuclei. The advent of highly polarized beams and targets and improvements in recoil polarization methods, as well as analysis and simulation techniques, have enabled us to study the static and dynamical properties of few-body systems with unprecedented precision. Recent experiments at Jefferson Lab and MAMI are presented and put into perspective of state-of-the art Faddeev calculations, with focus on the ${ }^{3} \mathrm{He}$ nucleus.
\end{abstract}

(c) (1) Copyright S. Širca.

This work is licensed under the Creative Commons

Attribution 4.0 International License.

Published by the SciPost Foundation.
Received 14-10-2019

Accepted 28-10-2019

Published 27-02-2020

doi:10.21468/SciPostPhysProc.3.048

\section{Contents}

1 Introduction 1

2 Double-spin asymmetries in ${ }^{3} \mathrm{He}$ breakup $\quad 2$

2.1 Deuteron channel 2

2.2 Proton channel 3

2.3 Neutron channel 5

2.4 Extension to triple polarization 5

$\begin{array}{lll}3 & \text { Single-spin asymmetries with transverse polarization } & 6\end{array}$

$\begin{array}{lll}4 & \text { Medium modification of elastic form-factors } & 7\end{array}$

5 Conclusion 9

$\begin{array}{lr}\text { References } & 9\end{array}$ 


\section{Introduction}

All modern electron-scattering experiments involving polarization degrees of freedom are being performed either at the Thomas Jefferson National Accelerator Facility (TJNAF or JLab) in Newport News, USA, or at the MAMI Accelerator Facility in Mainz, Germany. In this paper we present the results of a select list of the most relevant recent experiments: TJNAF E05-102, dedicated to the measurement of double-spin asymmetries in the ${ }^{3} \mathrm{He}\left(\vec{e}, e^{\prime} d\right) p,{ }^{3} \mathrm{He}\left(\vec{e}, e^{\prime} p\right) d$ and ${ }^{3} \mathrm{He}\left(\vec{e}, e^{\prime} p\right) p n$ processes in the quasi-elastic region; the TJNAF E05-015, whose main goal was the determination of target single-spin asymmetry in the quasi-elastic ${ }^{3} \mathrm{He}^{\uparrow}\left(\mathrm{e}, \mathrm{e}^{\prime}\right)$ process; the TJNAF E08-005 in which we studied target single-spin asymmetries in quasi-elastic ${ }^{3} \mathrm{He}^{\uparrow}\left(e, e^{\prime} n\right)$ and double-spin asymmetries in quasi-elastic ${ }^{3} \mathrm{He}\left(\overrightarrow{\mathrm{e}}, \mathrm{e}^{\prime} \mathrm{n}\right)$; and the MAMI Project 'N' with the first-ever attempt to investigate the triple-polarized process ${ }^{3} \mathrm{He}\left(\overrightarrow{\mathrm{e}}, \mathrm{e}^{\prime} \overrightarrow{\mathrm{p}}\right)$ in order to study the spin-dependent distribution of polarized pd clusters in ${ }^{3} \mathrm{He}$. The ${ }^{3} \mathrm{He}$ nucleus is indeed the playground for tests of few-body nuclear dynamics [1,2], but lately ${ }^{2} \mathrm{H}$ and ${ }^{12} \mathrm{C}$ have also received renewed attention due to their relevance for the study of medium modifications of elastic form-factors of nucleons; here we outline our efforts to determine the single-spin asymmetries in ${ }^{12} \mathrm{C}\left(\mathrm{e}^{\uparrow}, \mathrm{e}^{\prime}\right)$ as well as recoil polarizations in ${ }^{2} \mathrm{H}\left(\overrightarrow{\mathrm{e}}, \mathrm{e}^{\prime} \overrightarrow{\mathrm{p}}\right)$ and ${ }^{12} \mathrm{C}\left(\overrightarrow{\mathrm{e}}, \mathrm{e}^{\prime} \overrightarrow{\mathrm{p}}\right)$ processes.

\section{Double-spin asymmetries in ${ }^{3} \mathrm{He}$ breakup}

A precise knowledge of ground-state structure of ${ }^{3} \mathrm{He}$ is needed to extract the information on the neutron from all types of exclusive or inclusive experiments on polarized ${ }^{3} \mathrm{He}$, for instance, measurements of $G_{\mathrm{E}}^{\mathrm{n}}, G_{\mathrm{M}}^{\mathrm{n}}, A_{1}^{\mathrm{n}}, g_{1}^{\mathrm{n}}, g_{2}^{\mathrm{n}}$ or determinations of the GDH sum rule. The ground state of ${ }^{3} \mathrm{He}$, however, is quite complex, with the dominant $S$-state configuration complicated by $D$ and $S^{\prime}$-states as well as a multitude of sub-leading Faddeev components [3]. Polarization observables turn out to be most sensitive to ${ }^{3} \mathrm{He}$ ground-state structure, and several stateof-the-art calculations have been confronted with the measurements of double-polarization observables in ${ }^{3} \mathrm{He}\left(\overrightarrow{\mathrm{e}}, \mathrm{e}^{\prime} \mathrm{d}\right),{ }^{3} \mathrm{He}\left(\overrightarrow{\mathrm{e}}, \mathrm{e}^{\prime} \mathrm{p}\right)$, and ${ }^{3} \mathrm{He}\left(\overrightarrow{\mathrm{e}}, \mathrm{e}^{\prime} \mathrm{n}\right)$ processes. These three channels are discussed separately in the following.

The differential cross-section for electron-induced deuteron knockout from a polarized ${ }^{3} \mathrm{He}$ is given by

$$
\frac{\mathrm{d} \sigma(h, \vec{S})}{\mathrm{d} \Omega_{\mathrm{e}} \mathrm{d} E_{\mathrm{e}} \mathrm{d} \Omega_{\mathrm{d}} \mathrm{d} p_{\mathrm{d}}}=\sigma_{0}\left[1+\vec{S} \cdot \vec{A}^{0}+h\left(A_{\mathrm{e}}+\vec{S} \cdot \vec{A}\right)\right],
$$

where $\sigma_{0}$ is the "unpolarized" cross-section, the nuclear spin vector $\vec{S}$ and the helicity of the electron beam is $h$. The measured beam-target asymmetry

$$
A\left(\theta^{*}, \phi^{*}\right)=\vec{S}\left(\theta^{*}, \phi^{*}\right) \cdot \vec{A}=\frac{\left[\mathrm{d} \sigma_{++}+\mathrm{d} \sigma_{--}\right]-\left[\mathrm{d} \sigma_{+-}+\mathrm{d} \sigma_{-+}\right]}{\left[\mathrm{d} \sigma_{++}+\mathrm{d} \sigma_{--}\right]+\left[\mathrm{d} \sigma_{+-}+\mathrm{d} \sigma_{-+}\right]}
$$

depends on the orientation of $\vec{S}$, specified by the angles $\theta^{*}$ and $\phi^{*}$ with respect to momentum transfer. The same formalism applies to the remaining two channels, with the particle subscript 'd' replaced by 'p' or 'n'.

\subsection{Deuteron channel}

The results on beam-target asymmetries in the deuteron channel have been described in detail in Ref. [4]. Figure 1 shows the $A\left(71^{\circ}, 0^{\circ}\right)$ and $A\left(160^{\circ}, 0^{\circ}\right)$ asymmetries as functions of missing momentum, $p_{\mathrm{m}}$, together with the calculations of the Vilnius (V; formerly Hannover/Lisbon) [5-8], the Krakow (K; formerly Bochum/Krakow) $[9,10]$ and Pisa (P) [11] groups. The K and 
V calculations are Faddeev calculations with a complete treatment of final-state interactions (FSI) and meson-exchange currents (MEC); they differ only in their choice of the nucleonnucleon potential (AV18 vs. charge-dependent Bonn, respectively) and the inclusion of the three-nucleon force (typically Urbana IX vs. $\Delta$ isobar as an active degree of freedom to provide the effective three-nucleon strength); the $\mathrm{V}$ calculations also include a point Coulomb interaction in the partial waves involving two charged baryons. The P calculations (not Faddeev but of equivalent precision) are based on variational pair-correlated hyper-spherical harmonic expansions to render the FSI, and also include MEC.

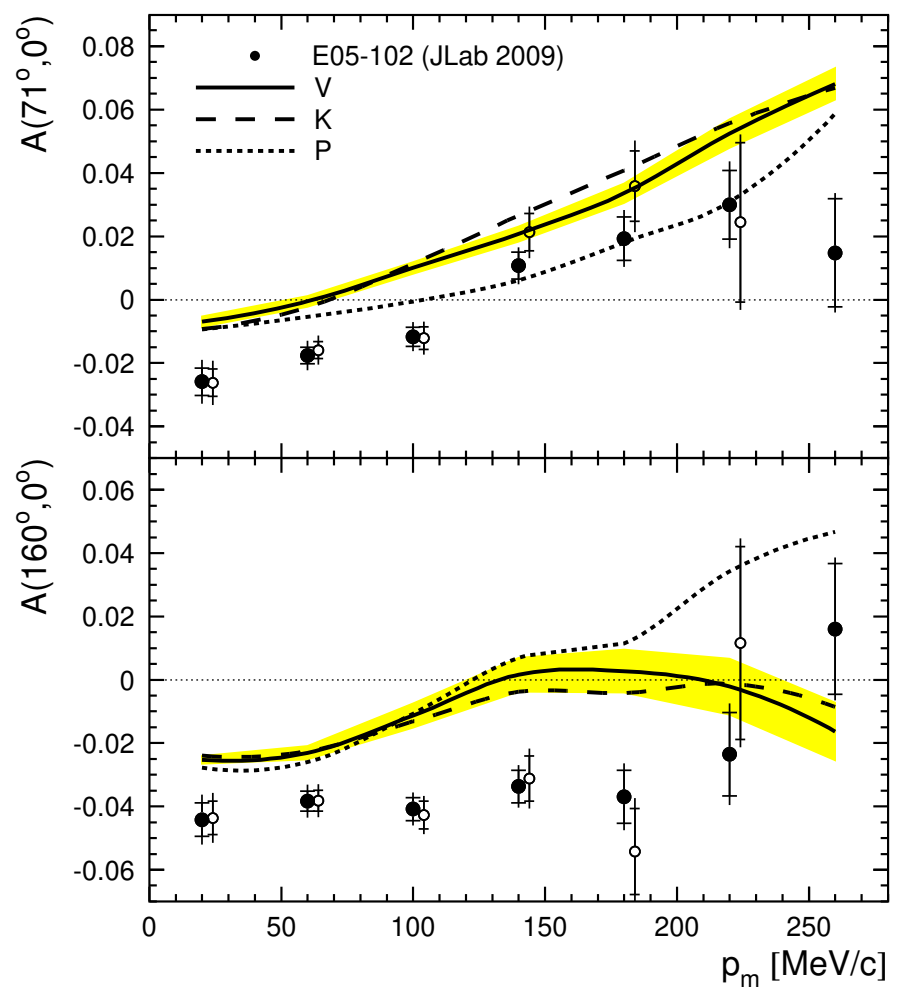

Figure 1: The measured asymmetries $A\left(71^{\circ}, 0^{\circ}\right)$ (top) and $A\left(160^{\circ}, 0^{\circ}\right)$ (bottom) in the quasi-elastic ${ }^{3} \mathrm{He}\left(\overrightarrow{\mathrm{e}}, \mathrm{e}^{\prime} \mathrm{d}\right)$ process as functions of missing momentum, compared to the acceptance-averaged V, K and P calculations (see text for notation). The empty symbols (shifted for clarity) denote the data with a cut on the quasi-elastic peak.

The measured $A\left(71^{\circ}, 0^{\circ}\right)$ asymmetry crosses zero at $p_{\mathrm{m}} \approx 130 \mathrm{MeV} / c$; this behavior is qualitatively mirrored by all calculations, although the zero crossing occurs at lower $p_{\mathrm{m}}$. Neither of the considered calculations reproduces the measured $A\left(160^{\circ}, 0^{\circ}\right)$ asymmetry (leveled at approximately $-4 \%$ throughout the $p_{\mathrm{m}}$ range). Modern theoretical treatments of the ${ }^{3} \mathrm{He}$ system are therefore only able to qualitatively account for the bulk of our data set; however, given the small magnitude of the asymmetries and the delicate interplay of their ingredients, the agreement can be considered to be satisfactory. The revealed deficiencies in the calculations indicate a need for further refinement in the treatment of two- and/or three-body dynamics, and this is an ongoing effort.

\subsection{Proton channel}

The results on beam-target asymmetries in the proton channel have been described in detail in Ref. [12]. In this channel, the energy resolution of the apparatus was insufficient to directly disentangle the two-body and three-body breakup contributions (2bbu and 3bbu, respectively). 
The individual asymmetries were therefore extracted by studying the missing-energy dependence of the asymmetries and relying on a Monte-Carlo simulation weighted with the corresponding unpolarized cross-sections. Figure 2 shows the $A\left(67^{\circ}, 0^{\circ}\right)$ asymmetry (top panel) and the $A\left(156^{\circ}, 0^{\circ}\right)$ asymmetry (bottom panel), together with the $\mathrm{K}$ and $\mathrm{V}$ calculations.
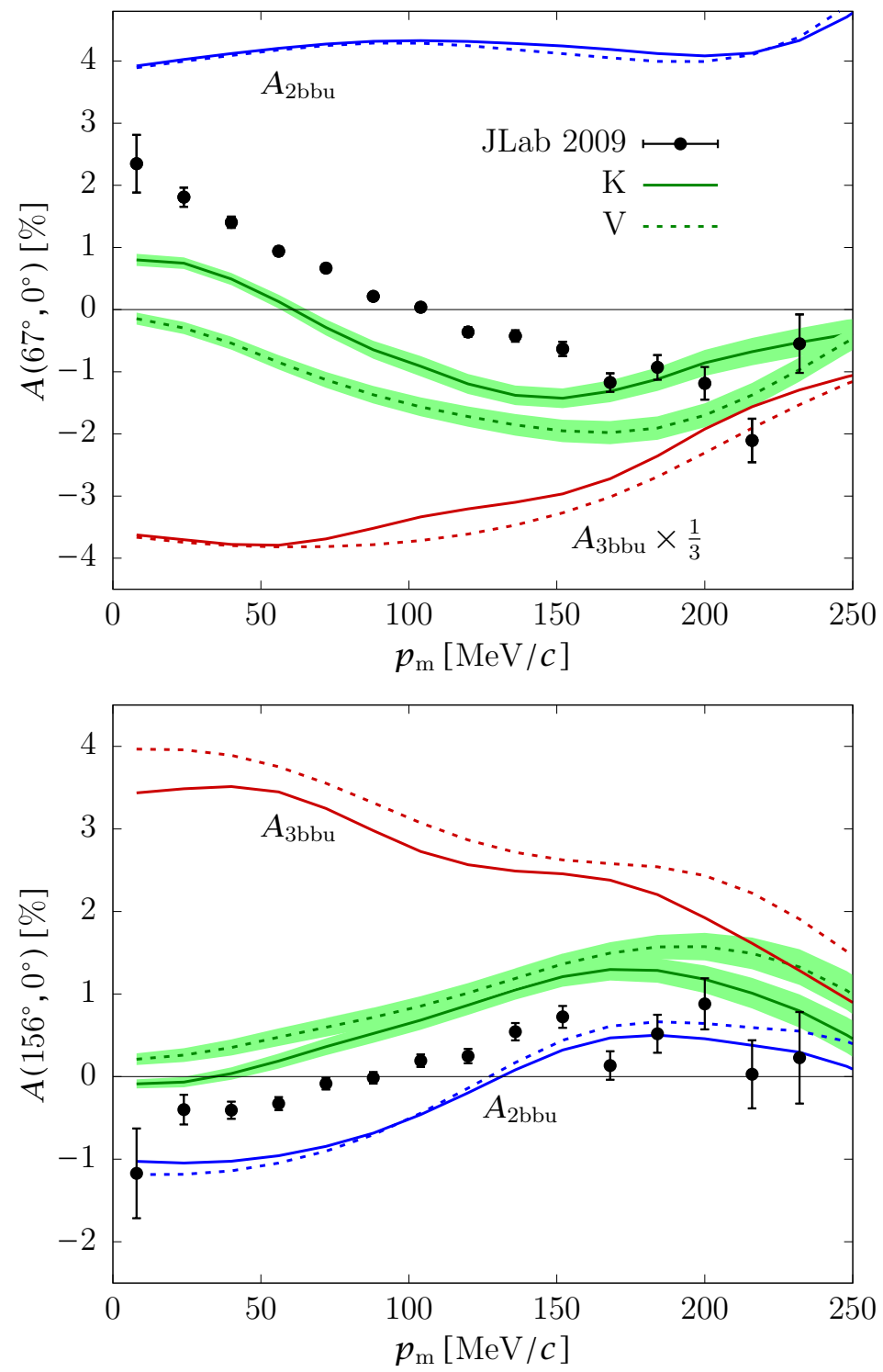

Figure 2: The measured asymmetries $A\left(67^{\circ}, 0^{\circ}\right)$ (top) and $A\left(156^{\circ}, 0^{\circ}\right)$ (bottom) in the quasi-elastic ${ }^{3} \mathrm{He}\left(\overrightarrow{\mathrm{e}}, \mathrm{e}^{\prime} \mathrm{p}\right)$ process as functions of missing momentum, compared to the acceptance-averaged $\mathrm{K}$ and $\mathrm{V}$ calculations (see text for notation). The calculated two-body and three-body breakup contributions are also shown.

The situation in the proton channel is similar to the one in the deuteron channel: the stateof-the-art theoretical approaches are able to approximately describe only the overall behavior of the data. We note, however, that the asymmetries are relatively small and therefore hard to reproduce, given the strong cancellations of the $2 \mathrm{bbu}$ and $3 \mathrm{bbu}$ contributions.

We have tried to assess the relative importance of $2 \mathrm{bbu}$ and $3 \mathrm{bbu}$ contributions by dividing the measured nuclear asymmetries by the asymmetries for elastic $\vec{e} \vec{p}$ scattering at the same value of $Q^{2}$; see Fig. 3. In the plane-wave approximation, the $2 \mathrm{bbu}$ ratio for the ${ }^{3} \mathrm{He}\left(\overrightarrow{\mathrm{e}}, \mathrm{e}^{\prime} \mathrm{p}\right)$ process at $p_{\mathrm{m}} \approx 0$ should be $-1 / 3$, corresponding to the effective polarization of the (almost free) proton inside the polarized ${ }^{3} \mathrm{He}$ nucleus, while the $3 \mathrm{bbu}$ ratio should vanish because the 
knockout process may involve any of the two oppositely polarized protons in the target. In the $2 \mathrm{bbu}$ case this is indeed what one observes - the experimental and the predicted ratios coincide - while in the 3bbu case the calculations and the data deviate significantly.

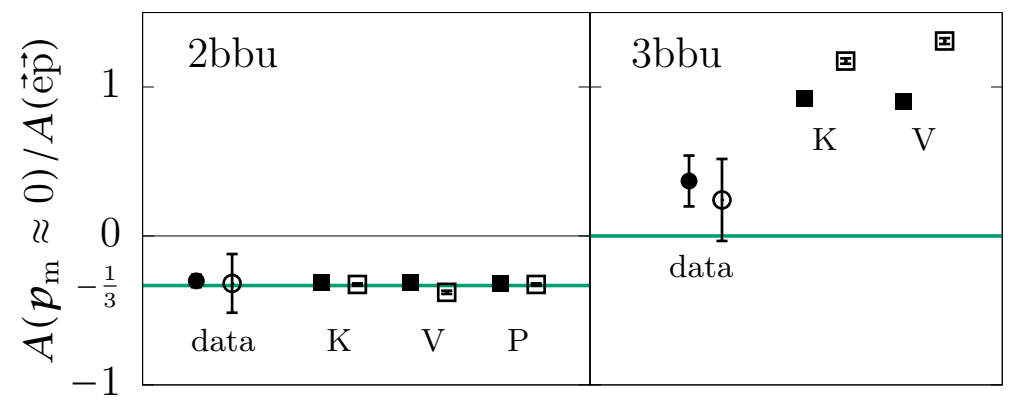

Figure 3: The $A\left(67^{\circ}, 0^{\circ}\right)$ (full symbols) and $A\left(156^{\circ}, 0^{\circ}\right)$ (empty symbols) asymmetries for $2 \mathrm{bbu}$ (left) and $3 \mathrm{bbu}$ (right) divided by the corresponding asymmetries for elastic $\overrightarrow{\mathrm{e}} \overrightarrow{\mathrm{p}}$ scattering at the same value of $Q^{2}$.

The measurements therefore indicate that the calculated 3bbu asymmetry is overestimated, and point to a mismatch between the true relativistic kinematics used in the analysis and non-relativistic spin-dependent nuclear dynamics employed in the calculations. This is also a matter of ongoing theoretical work; in particular, one would wish to verify whether consistent chiral two-nucleon and three-nucleon interactions with chiral two-nucleon and three-nucleon contributions in the electromagnetic current operator could provide a viable solution.

The measurements presented in Subsections 2.1 and 2.2 have been performed at momentum transfers of $Q^{2} \approx 0.25 \mathrm{GeV}^{2}$. High-statistics data in both channels are available also at $Q \approx 0.35 \mathrm{GeV}^{2}$. The analysis of this data set is work in progress; one of the obstacles is the lack of reliable Faddeev calculations in this momentum range (large relative kinetic energies of the outgoing particles).

\subsection{Neutron channel}

As part of the BigFamily group of experiments performed at TJNAF in 2009, we have also acquired precise data for the ${ }^{3} \mathrm{He}\left(\overrightarrow{\mathrm{e}}, \mathrm{e}^{\prime} \mathrm{n}\right)$ process in quasi-elastic kinematics at $Q^{2} \approx 0.5 \mathrm{GeV}^{2}$ and $Q^{2} \approx 0.95 \mathrm{GeV}^{2}$. The analysis of this data set is ongoing.

\subsection{Extension to triple polarization}

In a novel type of experiment, the A1 Collaboration at MAMI has also performed a measurement of proton knockout from ${ }^{3} \mathrm{He}$ using polarized electrons, polarized target, and detecting the polarization of the ejected protons, i. e. ${ }^{3} \mathrm{He}\left(\overrightarrow{\mathrm{e}}, \mathrm{e}^{\prime} \overrightarrow{\mathrm{p}}\right)$. This process offers a tool to study spin-dependent momentum distributions of $\vec{p} \vec{d}$ clusters in polarized ${ }^{3} \mathrm{He}$ [9]. In this formalism the yields $\mathcal{Y}$ in a specific target-ejectile spin configuration ( $M$ and $M_{\mathrm{d}} / m$, respectively) are related to the matrix elements

$$
N_{\mu}=\left\langle\Psi_{\mathrm{pd}}^{(-)} M_{\mathrm{d}} m\left|\widehat{j}_{\mu}(\vec{q})\right| \Psi M\right\rangle
$$

for the transition between the ground state, $\Psi$, and the final polarized pd pair, $\Psi_{\mathrm{pd}}$, through

$$
\begin{aligned}
& \mathcal{Y}\left(M=\frac{1}{2}, M_{\mathrm{d}}=0, m=+\frac{1}{2}\right) \propto\left|N_{-1}\left(\frac{1}{2}, 0,-\frac{1}{2}\right)\right|^{2}, \\
& \mathcal{Y}\left(M=\frac{1}{2}, M_{\mathrm{d}}=1, m=-\frac{1}{2}\right) \propto\left|N_{+1}\left(\frac{1}{2}, 1,+\frac{1}{2}\right)\right|^{2} .
\end{aligned}
$$


What is measured is the asymmetry

$$
A=\frac{\mathcal{Y}(1 / 2,0,1 / 2)-\mathcal{Y}(1 / 2,1,-1 / 2)}{\mathcal{Y}(1 / 2,0,1 / 2)+\mathcal{Y}(1 / 2,1,-1 / 2)} .
$$

The first result [13] is shown in Fig. 4.

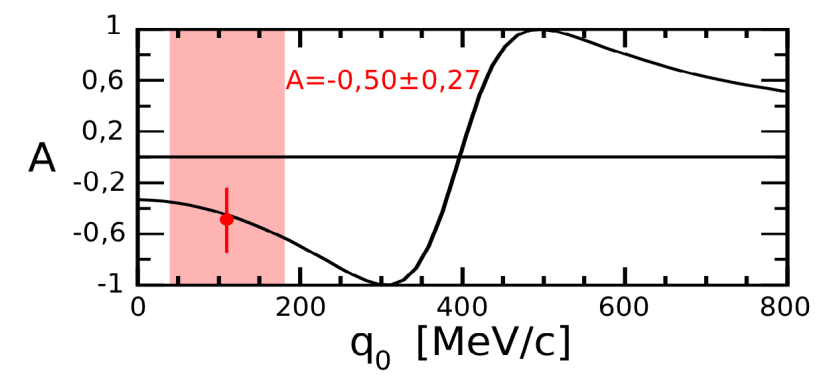

Figure 4: The asymmetry $A$ (defined by Eq. (1)) in the ${ }^{3} \overrightarrow{\mathrm{He}}\left(\overrightarrow{\mathrm{e}}, \mathrm{e}^{\prime} \overrightarrow{\mathrm{p}}\right)$ process.

Although this was was a pioneering study plagued by large statistical errors, it has shown that polarized ${ }^{3} \mathrm{He}$ can be used not only as an effective polarized neutron target, but also by simultaneous detection of the deuteron - as a polarized proton target. More theoretical work and more favorable experimental conditions are needed to further pursue this research.

\section{Single-spin asymmetries with transverse polarization}

The common denominator of electron scattering experiments on transversely polarized nuclear targets is their sensitivity to two-photon $(2 \gamma)$ exchange processes. One can investigate both inclusive or exclusive channels, and the key observable is the asymmetry

$$
A_{y}=\frac{\sigma^{\uparrow}-\sigma^{\downarrow}}{\sigma^{\uparrow}+\sigma^{\downarrow}} \propto \vec{S} \cdot\left(\vec{k} \times \vec{k}^{\prime}\right)
$$

where $\vec{S}$ is the target spin vector and $\vec{k}\left(\vec{k}^{\prime}\right)$ are the momenta of incoming (outgoing) electrons. This asymmetry is proportional to the $\operatorname{Im}\left\{T_{1 \gamma} T_{2 \gamma}^{*}\right\}$ interference. Assuming $T$-invariance, $A_{y}$ should vanish in the Born approximation, thus any deviation of $A_{y}$ from zero is indicative of $2 \gamma$ effects and becomes relevant e. g. for the extraction of elastic form-factor ratios, $G_{\mathrm{E}}^{\mathrm{p}} / G_{\mathrm{M}}^{\mathrm{p}}$, from polarimetry experiments, or determinations of generalized parton distributions (GPDs).

Some data on $A_{y}$ for the proton exist, but until recently there was no measurement of comparable precision on the neutron. The E05-015 experiment at TJNAF [14] yielded the first results on the target-normal single-spin asymmetry $A_{y}^{\mathrm{n}}$ from the inclusive ${ }^{3} \mathrm{He}^{\uparrow}\left(\mathrm{e}, \mathrm{e}^{\prime}\right)$ process with an uncertainty several times better than previous proton data. The results are shown in Fig. 5. The asymmetry is clearly non-zero and negative. In particular, at the highest measured $Q^{2}$ it agrees well with a prediction based on $2 \gamma$-exchange involving a model based on GPDs and therefore provides a new and independent constraint on these distributions.

The target-normal single-spin asymmetry has also been measured in the exclusive ${ }^{3} \mathrm{He}^{\uparrow}\left(\mathrm{e}, \mathrm{e}^{\prime} \mathrm{n}\right)$ reaction at $0.4 \leq Q^{2} \leq 1.0 \mathrm{GeV}^{2}$ [15]. This process constitutes and ideal probe of FSI and MEC: it should vanish in the plane-wave impulse approximation and is expected to fall off rapidly with increasing $Q^{2}$. The results shown in Fig. 6 confirm this expectation.

The kinematic reach of the presented measurements extends above the range of validity of Faddeev calculations (approximately up to $0.4 \mathrm{GeV}^{2}$ ) which lack relativistic effects, thus our data can not at present be analyzed by using these approaches. Still, it is remains clear that almost up to $1 \mathrm{GeV}^{2}$ any extraction of a neutron quantity from scattering on polarized ${ }^{3} \mathrm{He}$, 


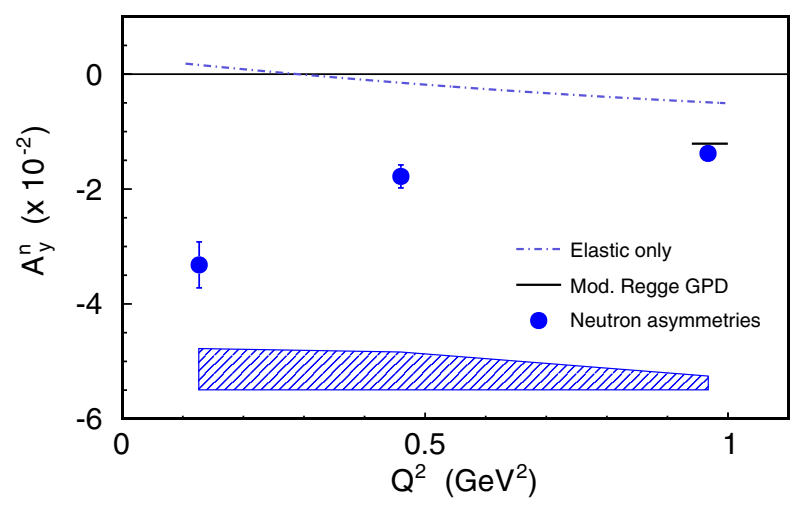

Figure 5: The target-normal single-spin asymmetry in inclusive ${ }^{3} \mathrm{He}^{\uparrow}\left(\mathrm{e}, \mathrm{e}^{\prime}\right)$.
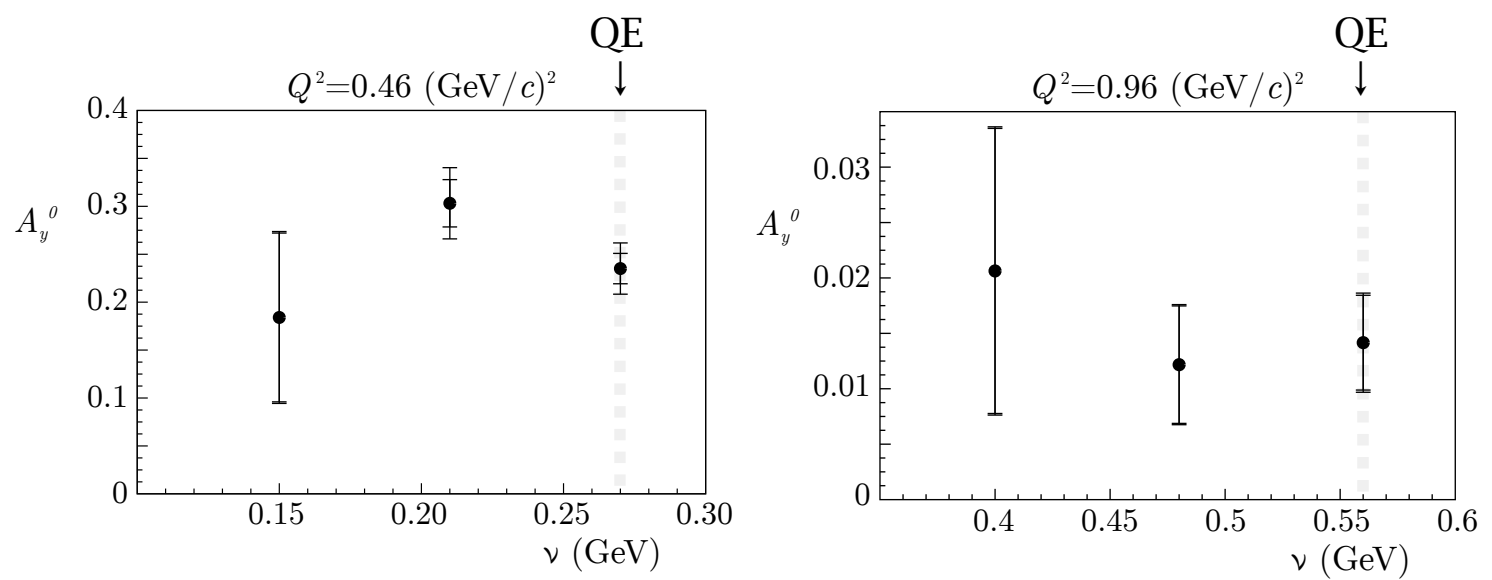

Figure 6: The target-normal single-spin asymmetry in the ${ }^{3} \mathrm{He}^{\uparrow}\left(\mathrm{e}, \mathrm{e}^{\prime} \mathrm{n}\right)$ process as functions of energy transfer, for two values of $Q^{2}$. The arrows indicate the location of the quasi-elastic peak.

for instance, neutron elastic form-factors, must account for the established effects of FSI and MEC. Only the highest $Q^{2}$ has been demonstrated to be free of them, although this region remains sensitive to $2 \gamma$-exchange. On the other hand, it is clear that for planned the $12 \mathrm{GeV}$ TJNAF experiments at high $Q^{2}$ the impulse approximation is justified.

Recently, and with similar physics goals in mind, single-spin asymmetries have also been measured in inclusive scattering of transversely polarized electrons on ${ }^{12} \mathrm{C}$ [16]. The asymmetry is again defined essentially as in Eq. (2), with $\vec{S}$ replaced by the electron spin vector, oriented perpendicularly to the scattering plane. One expects that $A_{y}$ for nuclear targets behaves as

$$
A_{y} \sim C_{0} \log \left(\frac{Q^{2}}{m_{\mathrm{e}}^{2} c^{2}}\right) \frac{F_{\text {Compton }}\left(Q^{2}\right)}{F_{\text {charge }}\left(Q^{2}\right)},
$$

with the $Q^{2}$-dependence (apart from the leading logarithmic factor) driven by the ratio of Compton to charge form-factors,

$$
\frac{F_{\text {Compton }}\left(Q^{2}\right)}{F_{\text {charge }}\left(Q^{2}\right)} \approx \exp \left[-4 Q^{2} / \mathrm{GeV}^{2}\right],
$$

roughly independent of the target nucleus. The aim of the MAMI experiment reported in Ref. [16] was to perform the first systematic study of the $Q^{2}$-dependence of the beam-normal single-spin asymmetry for a light nucleus. The study has shown that the assumption of the 
leading-log dominance and the independence of the $F_{\text {Compton }}\left(Q^{2}\right) / F_{\text {charge }}\left(Q^{2}\right)$ on the target nucleus may be too restrictive, and that even larger disagreements between the data and the calculations may be expected for heavier nuclei.

\section{Medium modification of elastic form-factors}

A number of models predict that the proton elastic form-factors are modified when the protons are embedded in a nucleus. The optimal way to test this hypothesis is to study the process of electron-induced quasi-elastic proton knockout from a nucleus with mass number $A$ by using polarized electrons and by detecting the polarization of the ejected protons, ${ }^{A} X\left(\overrightarrow{\mathrm{e}}, \mathrm{e}^{\prime} \overrightarrow{\mathrm{p}}\right)^{A-1} \mathrm{X}$. The embedding effect is thought to be $Q^{2}$ - and density-dependent: for example, protons in $p$ and $s$ shells of ${ }^{12} \mathrm{C}$ reside in local densities which differ roughly by a factor of two, and this is expected to bring about a medium modification of their form-factors at the level of a few percent [17]. The best current approach is to use polarimetry techniques to extract the proton polarization components $P_{x}^{\prime}$ and $P_{z}^{\prime}$ which, for a free proton, can be related to its form-factor ratio by

$$
\frac{G_{E}^{\mathrm{p}}}{G_{M}^{\mathrm{p}}}=-\left(\frac{P_{x}^{\prime}}{P_{z}^{\prime}}\right)_{\mathrm{p}} \frac{E_{\mathrm{e}}+E_{\mathrm{e}}^{\prime}}{2 M_{\mathrm{p}}} \tan \frac{\theta_{\mathrm{e}}}{2} .
$$

One then measures these two components for the nucleus $A$ and forms the "ratio of ratios",

$$
\left(\frac{P_{x}^{\prime}}{P_{z}^{\prime}}\right)_{A} /\left(\frac{P_{x}^{\prime}}{P_{z}^{\prime}}\right)_{\mathrm{p}},
$$

which can be studied as a function of $p_{\mathrm{m}}$, but a more natural independent variable is virtuality, a measure of "off-shellness" of the proton prior to its ejection. It can be defined somewhat arbitrarily, e. g. like

$$
v=p^{2}-m_{\mathrm{p}}^{2},
$$

but a more informed choice, taking into account the motion of the proton inside the nucleus, is

$$
v=\left(m_{A}-\sqrt{m_{A-1}^{2}+p_{\mathrm{m}}^{2}}\right)^{2}-p_{\mathrm{m}}^{2}-m_{\mathrm{p}}^{2} .
$$

The ratios (3) for three nuclei $\left({ }^{2} \mathrm{H},{ }^{4} \mathrm{He}\right.$ and ${ }^{12} \mathrm{C}$ ) measured at TJNAF and MAMI (see [18] and references therein), shown as functions of $v$, are shown in Fig. 7.

The most striking characteristic of the plot is the (approximate) independence of the double ratio on $A$ (on the nucleus), that is, a sort of universality. Clearly the double ratio changes substantially over the covered virtuality range, but from theoretical studies [19] it is also understood that a large (and likely dominant) contribution to this effect is due to FSI and the wave-function of the proton inside the nucleus. This makes the nuclear effects difficult to disentangle from the genuine medium form-factor modifications (if they exist). A new experiment at TJNAF has been proposed [20] to address the relative importance of these competing mechanisms at high $Q^{2}$. Further measurements at MAMI with other nuclei are also planned.

\section{Conclusion}

Electron scattering involving polarization degrees of freedom is an extremely sensitive tool to probe the electro-magnetic and spin structure of nuclei, with a much better selectivity and model-testing capability than "traditional" (unpolarized) cross-section measurements. Its use 


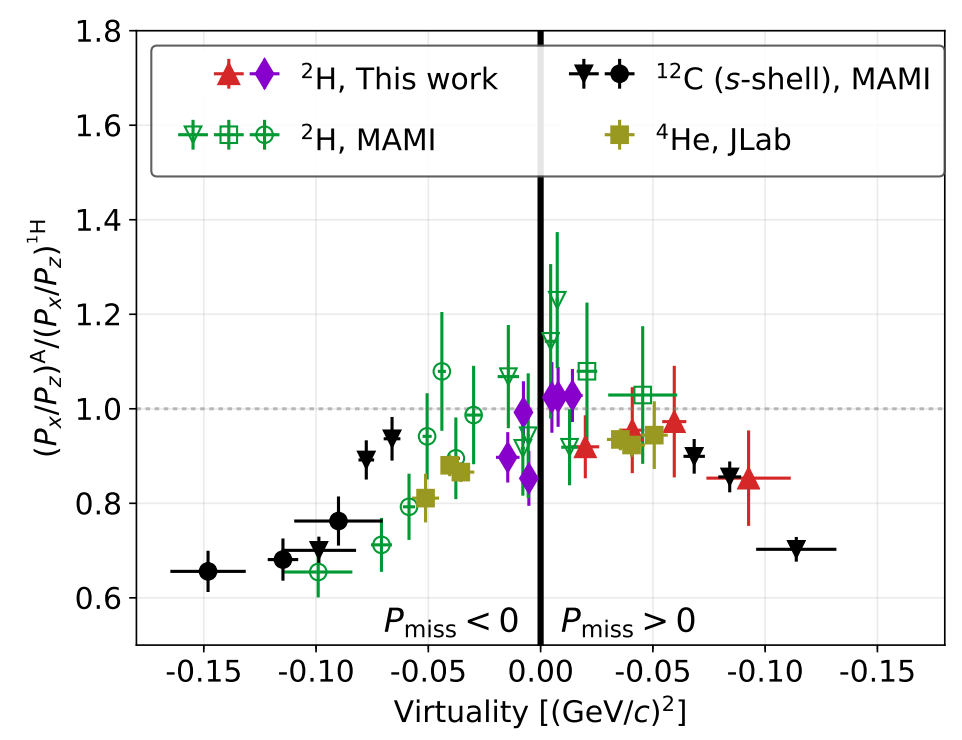

Figure 7: The double ratio (3) for $\left({ }^{2} \mathrm{H},{ }^{4} \mathrm{He}\right.$ and $\left.{ }^{12} \mathrm{C}\right)$ as a function of virtuality. As virtuality is always negative, the two branches of the plot are defined by the sign of missing momentum (its direction relative to momentum transfer).

in recent years has yielded important new results on the dynamics of breakup processes of ${ }^{3} \mathrm{He}$, on two-photon exchange effects, and (possible) medium modifications of proton elastic form-factors.

\section{Acknowledgments}

We thank the Jefferson Lab Hall A and Accelerator Operations technical staff for their outstanding support.

Funding information This work was supported in part by the National Science Foundation and the U.S. Department of Energy. Jefferson Science Associates, LLC, operates Jefferson Lab for the U.S. DOE under U.S. DOE contract DE-AC05-06OR23177. This work was supported in part by the Slovenian Research Agency (research core funding No. P1-0102). This work is a part of the LENPIC project and was supported by the Polish National Science Centre under Grants No. 2016/22/M/ST2/00173 and 2016/21/D/ST2/01120. The numerical calculations of the Krakow group were partially performed on the supercomputer cluster of the JSC, Jülich, Germany.

\section{References}

[1] W. Glöckle, J. Golak, R. Skibiński, H. Witała, H. Kamada and A. Nogga, Electron scattering on ${ }^{3} \mathrm{He}-$ A playground to test nuclear dynamics, Eur. Phys. J. A 21, 335 (2004), doi:10.1140/epja/i2004-10001-5.

[2] J. Golak, R. Skibiński, H. Witała, W. Glöckle, A. Nogga and H. Kamada, Electron and photon scattering on three-nucleon bound states, Phys. Rep. 415, 89 (2005), doi:10.1016/j.physrep.2005.04.005. 
[3] B. Blankleider and R. M. Woloshyn, Quasi-elastic scattering of polarized electrons on polarized ${ }^{3} \mathrm{He}$, Phys. Rev. C 29, 538 (1984), doi:10.1103/PhysRevC.29.538.

[4] M. Mihovilovič et al. (Hall A Collaboration), Measurement of double-polarization asymmetries in the quasielastic ${ }^{3} \vec{H} e\left(\vec{e}, e^{\prime} d\right)$ process, Phys. Rev. Lett. 113, 232505 (2014), doi:10.1103/PhysRevLett.113.232505.

[5] A. Deltuva, L. P. Yuan, J. Adam Jr., A. C. Fonseca and P. U. Sauer, Trinucleon photonuclear reactions with $\Delta$-isobar excitation: Processes below pion-production threshold, Phys. Rev. C 69, 034004 (2004), doi:10.1103/PhysRevC.69.034004.

[6] A. Deltuva, L. P. Yuan, J. Adam Jr. and P. U. Sauer, Three-body electrodisintegration of the three-nucleon bound state with $\Delta$-isobar excitation: Processes below pion-production threshold, Phys. Rev. C 70, 034004 (2004), doi:10.1103/PhysRevC.70.034004.

[7] A. Deltuva, A. C. Fonseca and P. U. Sauer, Momentum-space treatment of the Coulomb interaction in three-nucleon reactions with two protons, Phys. Rev. C 71, 054005 (2005), doi:10.1103/PhysRevC.71.054005.

[8] A. Deltuva, A. C. Fonseca and P. U. Sauer, Momentum-space description of three-nucleon breakup reactions including the Coulomb interaction, Phys. Rev. C 72, 054004 (2005), doi:10.1103/PhysRevC.72.054004.

[9] J. Golak, W. Glöckle, H. Kamada, H. Witała, R. Skibiński and A. Nogga, Spin dependent momentum distributions of proton-deuteron clusters in ${ }^{3} \mathrm{He}$ from electron scattering on polarized ${ }^{3} \mathrm{He}$ : Theoretical predictions, Phys. Rev. C 65, 064004 (2002), doi:10.1103/PhysRevC.65.064004.

[10] J. Golak, R. Skibiński, H. Witała, W. Glöckle, A. Nogga and H. Kamada, Proton polarizations in polarized ${ }^{3} \mathrm{He}$ studied with the ${ }^{3} \overrightarrow{H e}\left(\vec{e}, e^{\prime} p\right) d$ and ${ }^{3} \vec{H} e\left(\vec{e}, e^{\prime} p\right) p n$ processes, Phys. Rev. C 72, 054005 (2005), doi:10.1103/PhysRevC.72.054005.

[11] L. Marcucci, M. Viviani, R. Schiavilla, A. Kievsky and S. Rosati, Electromagnetic structure of $A=2$ and 3 nuclei and the nuclear current operator, Phys. Rev. C 72, 014001 (2005), doi:10.1103/PhysRevC.72.014001.

[12] M. Mihovilovič et al. (Hall A Collaboration), Measurement of double-polarization asymmetries in the quasi-elastic ${ }^{3} \overrightarrow{H e}\left(\vec{e}, e^{\prime} p\right)$ process, Phys. Lett. B 788, 117 (2019), doi:10.1016/j.physletb.2018.10.063.

[13] M. Weinriefer, Untersuchung der Kernstruktur von ${ }^{3} \mathrm{He}$ mittels Polarisationsobservablen, Doctoral Thesis, Johannes Gutenberg-Universität Mainz (2011).

[14] Y. Zhang et al. (Hall A Collaboration), Measurement of the target-normal single-spin asymmetry in quasielastic scattering from the reaction ${ }^{3} \mathrm{He}^{\uparrow}$ (e,e'), Phys. Rev. Lett. 115, 172502 (2015), doi:10.1103/PhysRevLett.115.172502.

[15] E. Long et al., Measurement of the single-spin asymmetry $A_{y}^{0}$ in quasi-elastic ${ }^{3} \mathrm{He}^{\uparrow}\left(e, e^{\prime} n\right)$ scattering at $0.4<Q^{2}<1.0 \mathrm{GeV} / \mathrm{c}^{2}$, Phys. Lett. B 797, 134875 (2019), doi:10.1016/j.physletb.2019.134875.

[16] A. Esser et al., First measurement of the $Q^{2}$ dependence of the beam-normal single spin asymmetry for elastic scattering off carbon, Phys. Rev. Lett. 121, 022503 (2018), doi:10.1103/PhysRevLett.121.022503. 
[17] G. Ron, W. Cosyn, E. Piasetzky, J. Ryckebusch and J. Lichtenstadt, Nuclear density dependence of in-medium polarization, Phys. Rev. C 87, 028202 (2013), doi:10.1103/PhysRevC.87.028202.

[18] S. Paul et al. (A1 Collaboration), Quasi-elastic polarization-transfer measurements on the deuteron in anti-parallel kinematics, Phys. Lett. B 795, 599 (2019), doi:10.1016/j.physletb.2019.07.002.

[19] S. Boffi, C. Giusti, F. D. Pacati and M. Radici, Nucleon recoil polarization in electromagnetic quasi-free knockout including two-body currents, Nucl. Phys. A 518, 639 (1990), doi:10.1016/0375-9474(90)90183-M.

[20] S. Strauch, E. Brash, G. Huber, R. Ransome, (co-spokespersons), Proton recoil polarization in the ${ }^{4} \mathrm{He}\left(\overrightarrow{\mathrm{e}}, \mathrm{e}^{\prime} \overrightarrow{\mathrm{p}}\right){ }^{3} \mathrm{H},{ }^{2} \mathrm{H}\left(\overrightarrow{\mathrm{e}}, \mathrm{e}^{\prime} \overrightarrow{\mathrm{p}}\right) \mathrm{n}$ and ${ }^{1} \mathrm{H}\left(\overrightarrow{\mathrm{e}}, \mathrm{e}^{\prime} \overrightarrow{\mathrm{p}}\right)$ reactions, TJNAF Experiment E1211-002. 